\title{
Right ventricular dysfunction and pulmonary hypertension in COVID-19: a meta-analysis of prevalence and its association with clinical outcome
}

Yudi Her Oktaviono ${ }^{1}$, Eka Prasetya Budi Mulia ${ }^{1}$, Kevin Luke², David Nugraha², Irma Maghfirah', Agus Subagjo ${ }^{1}$

${ }^{1}$ Department of Cardiology and Vascular Medicine, Faculty of Medicine, Universitas Airlangga - Dr. Soetomo General Hospital, Surabaya, Indonesia

${ }^{2}$ Faculty of Medicine, Universitas Airlangga, Surabaya, Indonesia

Submitted: 26 January 2021; Accepted: 3 May 2021

Online publication: 5 May 2021

Arch Med Sci 2022; 18 (5): 1169-1180

DOI: https//doi.org/10.5114/aoms/136342

Copyright @ 2022 Termedia \& Banach

\section{Abstract}

Introduction: Rapid spread of COVID-19 has caused detrimental effects globally. Involvement of the ACE2 receptor has identified COVID-19 as a multi-organ disease. Preliminary studies have provided evidence that cardiac involvement, including right ventricular dysfunction (RVD) and pulmonary hypertension (PH), were found in COVID-19 cases, even in the non-advanced stage. This meta-analysis aims to analyze the prevalence of RVD and $\mathrm{PH}$, and their association with COVID-19 clinical outcome.

Material and methods: A systematic data search was conducted through PubMed, medRxiv, ProQuest, Science Direct, and Scopus databases using constructed keywords based on MeSH terms. Any outcomes regarding mortality, severity, ICU admission, and mechanical ventilation usage were analyzed using RevMan v.5.4 and Stata v.16.

Results: A total of 16 eligible studies (1,728 patients) were included. Pooled prevalence of RVD in COVID- 19 was $19 \%$ (95\% Cl: $13-25 \%)$, and PH was $22 \%$ (95\% Cl: $14-31 \%)$. RVD was associated with increased mortality $(\mathrm{OR}=2.98$ (95\% Cl: 1.50-5.89), $p=0.002)$, severity $(\mathrm{OR}=3.61(95 \% \mathrm{Cl}: 2.05-6.35)$, $p<0.001)$, ICU admission ( $\mathrm{OR}=1.70(95 \% \mathrm{Cl}: 1.12-2.56), p=0.01)$, and mechanical ventilation (MV) usage ( $\mathrm{OR}=1.60(95 \% \mathrm{Cl}: 1.14-2.25), p=0.007)$. $\mathrm{PH}$ was also associated with increased mortality $(\mathrm{OR}=5.42(95 \% \mathrm{Cl}: 2.66-$ $11.060, p<0.001)$, severity (OR $=5.74$ (95\% Cl: 2.28-14.49), $p<0.001)$, and ICU admission (OR $=12.83(95 \% \mathrm{Cl}: 3.55-46.41), p<0.001)$.

Conclusions: RVD and PH were prevalent in COVID-19 and associated with mortality, severity, ICU admission, and MV usage in COVID-19 patients. Bedside echocardiography examination could be considered as a novel risk stratification tool in COVID-19.

Key words: COVID-19, right ventricular dysfunction, pulmonary hypertension, prevalence, outcome.

\section{Introduction}

The rapid spread of coronavirus disease 2019 (COVID-19) since December 2019 has caused detrimental effects globally. More than 50 million people had been infected with COVID-19, causing more than 1.2 million deaths worldwide [1]. SARS-CoV-2 was identified as the culprit and utilizes the angiotensin-converting enzyme 2 (ACE2) receptor for

\author{
Corresponding author: \\ Yudi Her Oktaviono \\ Department of Cardiology \\ and Vascular Medicine \\ Faculty of Medicine \\ Universitas Airlangga - \\ Dr. Soetomo \\ General Hospital \\ Surabaya, Indonesia \\ E-mail:yoktaviono@gmail. \\ com
}


host entry. The ACE2 receptor is widely expressed in the lungs, heart, vascular, and intestine. Hence, COVID-19 is not limited to lung disease but is a multi-organ disease. A post-mortem study of 32 patients revealed that COVID-19 caused thromboembolic injuries in the lung, heart, liver, kidney, and hematopoietic system [2].

Since ACE2 is closely related to the cardiovascular system, the impact of COVID-19 on this system is inevitable. Pre-existing cardiovascular disease comorbidity was associated with a higher case fatality rate and poor prognosis [3]. A previous study provided evidence that severe cardiac dysfunction, injury, and elevation of cardiac markers were found in COVID-19 cases [4]. On the other hand, one meta-analysis demonstrated that cardiac injury in COVID-19 is associated with a higher risk of severe disease (13.81-fold), ICU admission (7.94fold), and mortality (7.95-fold), respectively [5]. Taken together, bidirectional interaction between COVID-19 and cardiac conditions that impair cardiac and lung functions was inevitable, especially the involvement of right ventricular dysfunction and pulmonary hypertension [6, 7].

The mechanisms behind this interaction are not yet established. Release of systemic cytokine, systemic inflammation, and a pro-thrombotic state may be responsible. Furthermore, hypoxic vasoconstriction of the pulmonary vasculature in COVID-19 patients could alter pulmonary hemodynamics, damaging lung tissue, which leads to pulmonary hypertension $(\mathrm{PH})$ and right ventricular dysfunction (RVD) $[8,9]$. One case series described 5 cases of acute cor-pulmonale in critically ill COVID-19 patients [10]. Acute respiratory distress syndrome (ARDS) has been frequently reported among critically ill COVID-19 patients, and elevated RV afterload could also lead to RV function impairment [11].

Preliminary pathological findings indicate alveolar septal thickening, lung edema, inflammatory infiltrates, and vascular congestion in the early stages of the disease. PH and secondary RVD may be determined by lung parenchymal disruption and altered pulmonary hemodynamics in patients with COVID-19, even in the non-advanced stage of the disease [7].

Interestingly, early echocardiographic studies regarding RVD and $\mathrm{PH}$ parameters in COVID-19 revealed their potential as novel risk-stratification markers in COVID-19 patients since they outperformed others risk factors [12, 13]. Despite their potential in COVID-19 risk stratification, to the best of our knowledge, no meta-analysis has evaluated clinical outcomes regarding RVD and $\mathrm{PH}$ in COVID-19.

Therefore, this study aims to analyze the prevalence of RVD and $\mathrm{PH}$ along with their association with COVID-19 clinical outcomes.

\section{Material and methods}

\section{Study design and search strategy}

This systematic review and meta-analysis were performed based on Preferred Reporting Items for Systematic Reviews and Meta-Analyses (PRISMA) guidelines. Initial title and abstract screening was performed in 5 online databases (PubMed, medRxiv, ProQuest, ScienceDirect, and Scopus) using "COVID-19", "right ventricular dysfunction", "cardiac function", "pulmonary hypertension", "echocardiography", "severity”, “death", "mechanical ventilation", and their synonyms as searching keywords. The search period was in November 2020. We included all studies that reported adult COVID-19 patients with any data regarding right ventricular dysfunction or pulmonary hypertension and clinical outcome. All types of criteria and definitions of RVD and PH were included in this study. Additionally, any transthoracic echocardiographic parameters for RV function and $\mathrm{PH}$ were also included. Studies with incomplete data and not written in English were excluded.

\section{Data extraction}

Three authors independently screened the relevance of titles and abstracts after removing duplicates. Studies that met inclusion criteria were then assessed for the full article and further reviewed before data extraction. Subsequently, the authors extracted the data, consisting of authors, year of publication, study design, location, peer-reviewed publication status, sample size, male percentage, mean age, comorbidities, RVD and PH definitions, echocardiography profile, and severity criteria in each comparison group. All extracted data were collected in a dedicated Excel spreadsheet.

\section{Outcome}

The primary outcome in our meta-analysis was the association of RVD and PH with in-hospital mortality from COVID-19. The secondary outcome was disease severity, ICU admission, or the need for mechanical ventilation. We defined disease severity criteria based on the World Health Organization (WHO) and the National Health Commission of the People's Republic of China [14]. If the study categorized severity into 3 or 4 groups, we combined the data between mild and moderate groups into one group as non-severe; and severe and critical groups into one group as severe. These outcomes were classified as RVD and non-RVD or $\mathrm{PH}$ and non- $\mathrm{PH}$. Additionally, echocardiographic parameters were also compared and classified based on each outcome, namely survivors and non-survivors; severe and non-severe, admitted 
and not admitted to ICU; use and non-use of mechanical ventilation.

\section{Quality assessment and publication bias}

Two authors independently assessed study methodological quality using the Newcastle-Ottawa Scale (NOS) for non-randomized studies. Grading of Recommendations Assessment, Development, and Evaluation (GRADE) methodology was used to assess the quality of the body of retrieved evidence (GRADEpro Guideline Development Tool [Software]. McMaster University, 2020). Egger's and Harbord's regression test was used to assess publication bias for continuous and binary endpoints, respectively. In addition, funnel plots were used to determine the symmetrical distribution of the effect size outcomes.

\section{Data analysis}

All analyses were performed using RevMan version 5.4 and Stata v.16. We used the Mantel-Haenszel formula for dichotomous variables to calculate the pooled odds ratios (ORs). A random-effect model was performed if there was heterogeneity $\left(I^{2}>50 \%\right)$. Otherwise, the fixed-effects Mantel-Haenszel model was used. The cause of heterogeneity was assessed using sensitivity analysis with the leave-one-out method. We also performed a meta-analysis using the mean difference (MD) for echocardiographic parameters of $\mathrm{RV}$ function and $\mathrm{PH}$. Mean and standard deviation were extrapolated from sample size, median, and interquartile range (IQR), according to Wan et al. [15] The average of mean and standard deviation between two groups was calculated using the formula in Table 7.7.a of the Cochrane Handbook [16]. Restricted maximum likelihood random-effects meta-regression was performed for age, sex, cardiovascular disease (CVD), hypertension (HTN), diabetes mellitus (DM), chronic kidney disease (CKD), chronic obstructive pulmonary disease (COPD), venous thromboembolism (VTE), and smoking. Statistical significance was defined by a $p$-value less than 0.05 , except for heterogeneity $(p<0.10)$.

\section{Results}

\section{Baseline characteristics and study selection}

We found 584 records from the PubMed, Science Direct, ProQuest, Scopus, and medRxiv databases, as shown in Figure 1. Seven additional records were acquired from other sources, and 503 records remained after duplicate removal. A sum of 453 records was removed after title and abstracts screening. Fifty full texts were then assessed for eligibility, and 34 articles were excluded because of an incorrect patient population $(n=3)$; no data of RVD/PH ( $n=13)$; no outcome of interest $(n=11)$; and irrelevant severity criteria/ group $(n=4)$. As a result, we included 16 eligible studies (1,728 patients) for analysis.

Baseline characteristics of the included studies are presented in Table I [17-28] and Supplementary

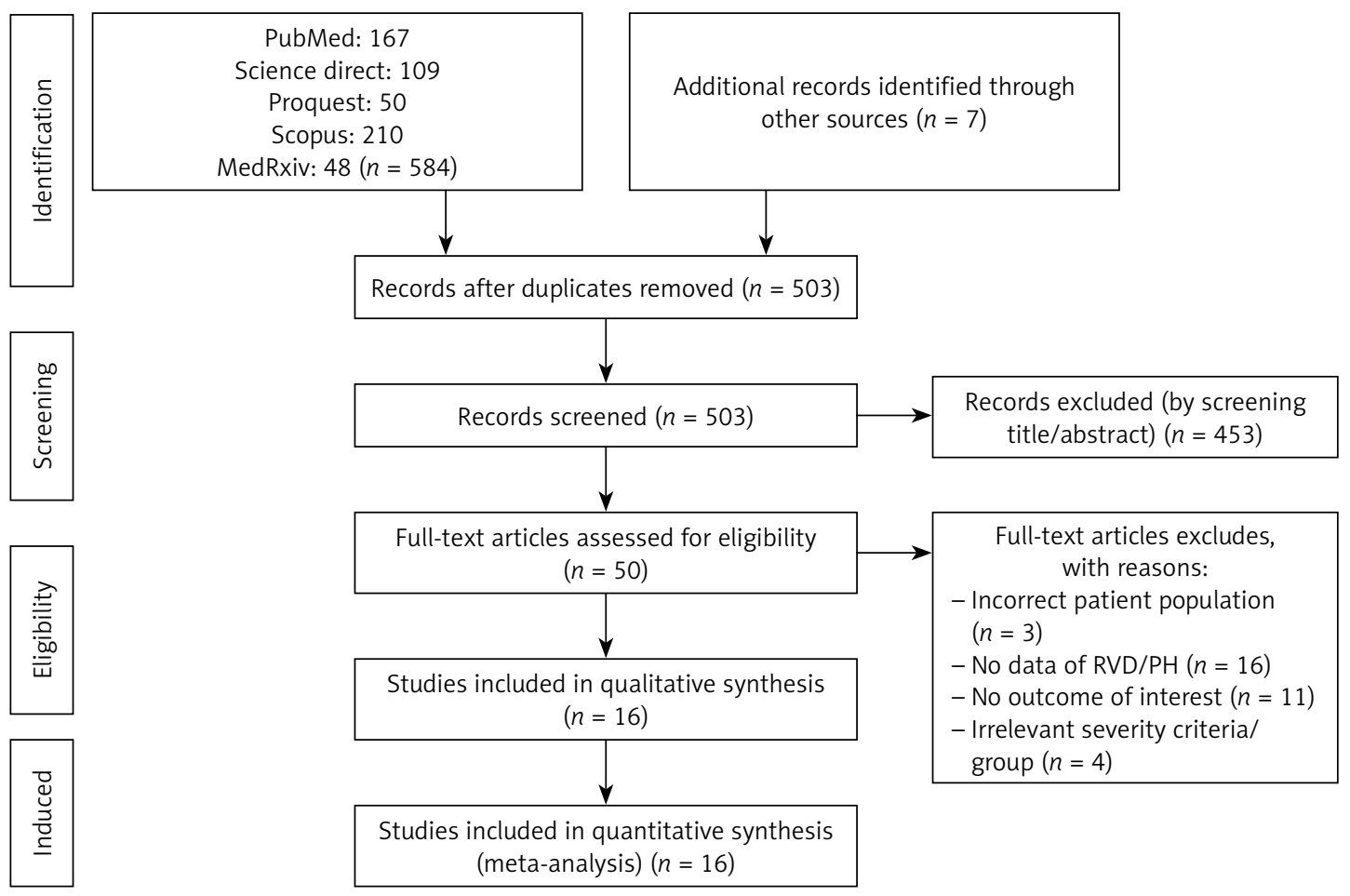

Figure 1. Study flow chart (as per PRISMA guideline) 


\begin{tabular}{|c|c|c|c|c|c|c|c|c|c|}
\hline 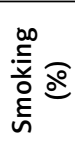 & $\stackrel{\frac{\pi}{c}}{\check{c}}$ & 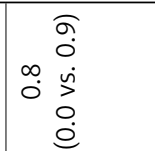 & 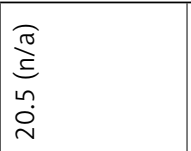 & $\frac{\sqrt{0}}{\stackrel{2}{n}}$ & 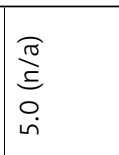 & $\begin{array}{l}\sqrt{0} \\
\stackrel{a}{a} \\
m \\
-1\end{array}$ & $\stackrel{\sigma}{\Sigma}$ & $\stackrel{\sigma}{\Omega}$ & $\stackrel{\sigma}{\Sigma}$ \\
\hline $\begin{array}{l}\text { o } \\
0 \\
0 \\
0\end{array}$ & 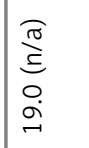 & $\stackrel{\pi}{\leq}$ & 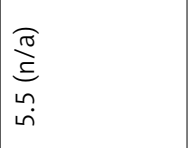 & 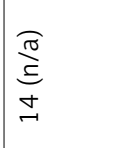 & 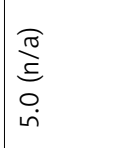 & 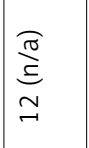 & 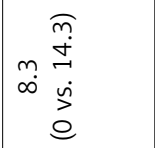 & 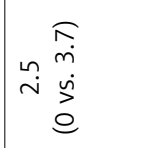 & 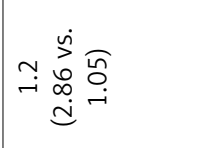 \\
\hline $\begin{array}{l}\overparen{o} \\
\stackrel{8}{0}\end{array}$ & 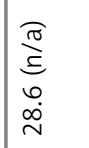 & 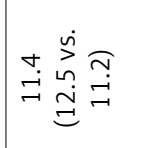 & 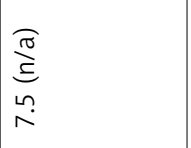 & $\begin{array}{l}\stackrel{\Xi}{\Xi} \\
\Xi \\
\exists\end{array}$ & 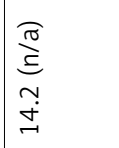 & 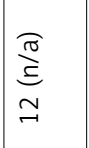 & $\begin{array}{rl} & 0 \\
r & \infty \\
0 & 0 \\
0 & \dot{1} \\
- & 0 \\
0\end{array}$ & 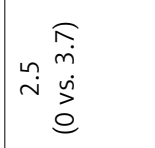 & 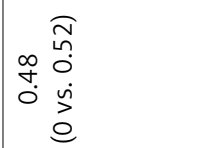 \\
\hline 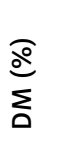 & 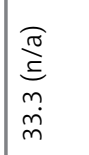 & 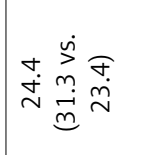 & 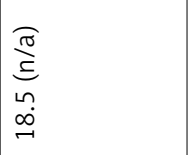 & 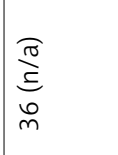 & 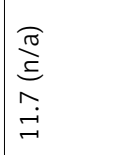 & $\begin{array}{l}\text { } \\
\stackrel{\sigma}{\Xi} \\
\tilde{m} \\
m\end{array}$ & 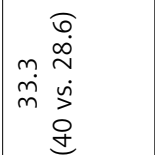 & 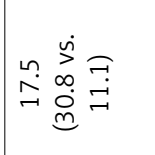 & 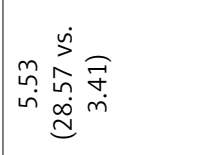 \\
\hline ְ̊ & $\stackrel{\pi}{\leq}$ & 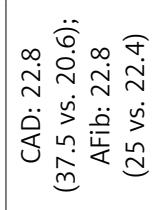 & 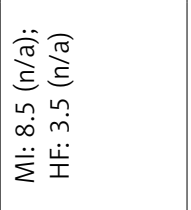 & 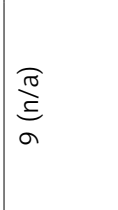 & 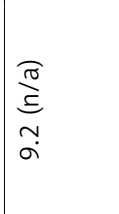 & 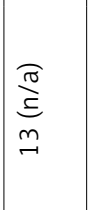 & 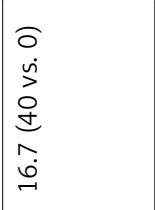 & $\begin{array}{l}0 \\
\dot{3} \\
0\end{array}$ & 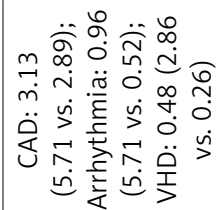 \\
\hline 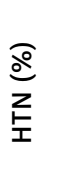 & 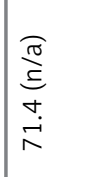 & 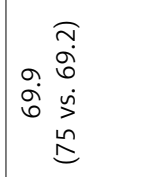 & 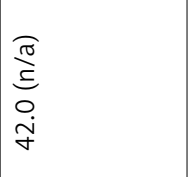 & 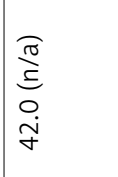 & 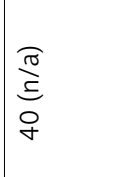 & 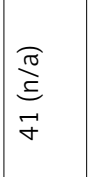 & 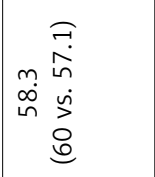 & 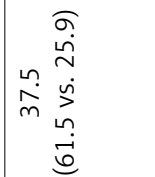 & 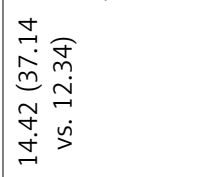 \\
\hline 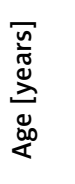 & 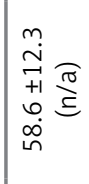 & 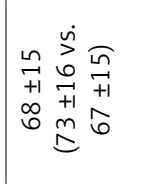 & 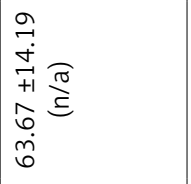 & 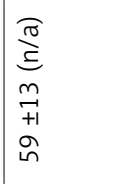 & 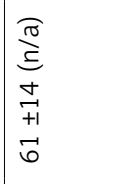 & 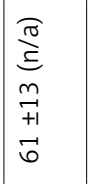 & 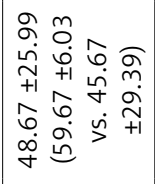 & 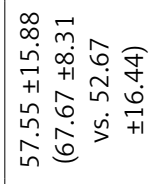 & 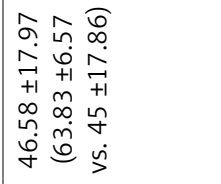 \\
\hline $\begin{array}{l}\frac{0}{0} \\
\frac{0}{20} \\
\frac{\pi}{2}\end{array}$ & 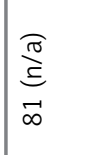 & 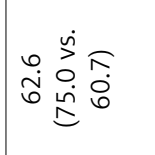 & 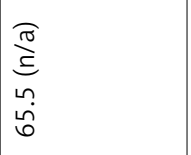 & 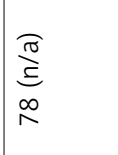 & $\begin{array}{l}\stackrel{\pi}{\Xi} \\
\stackrel{\infty}{\infty} \\
\stackrel{+}{+}\end{array}$ & 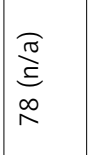 & 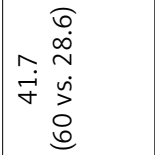 & 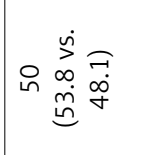 & 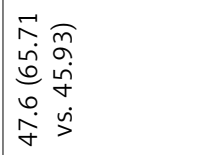 \\
\hline 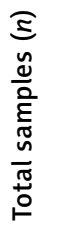 & $\begin{array}{l}\underset{\hat{m}}{\sim} \\
\dot{s} \\
\dot{\infty} \\
\infty \\
\vec{N}\end{array}$ & 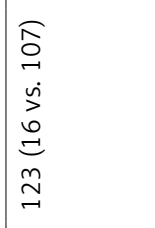 & 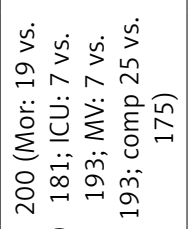 & 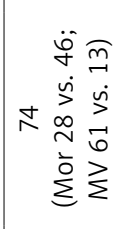 & 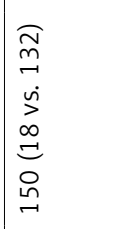 & $\begin{array}{l}0 \\
0 \\
0 \\
ن \\
0 \\
0 \\
0 \\
0 \\
0 \\
0 \\
-1\end{array}$ & 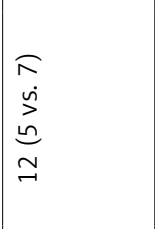 & 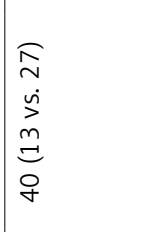 & 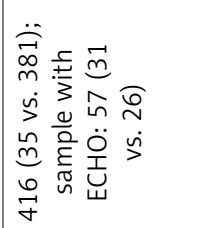 \\
\hline $\begin{array}{l}\text {.o. } \\
\text { 임 }\end{array}$ & $\stackrel{\sigma}{\Sigma}$ & 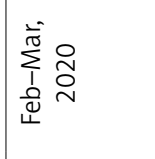 & 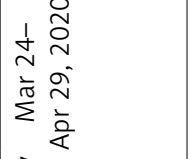 & 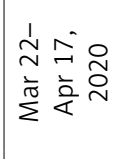 & 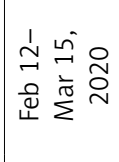 & $\left|\begin{array}{ll}1 & 0 \\
b & 0 \\
0 & 0 \\
1 & 0 \\
i \pi & 0 \\
\sum & \frac{\pi}{2}\end{array}\right|$ & $\stackrel{\pi}{\Sigma}$ & 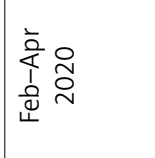 & 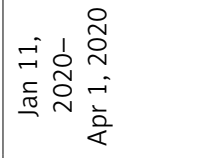 \\
\hline 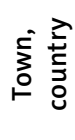 & \begin{tabular}{|l} 
ते \\
है \\
है \\
J
\end{tabular} & 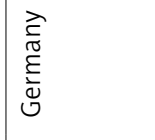 & 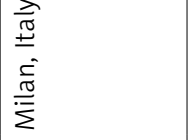 & 兰 & 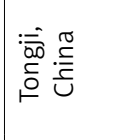 & 弚 & 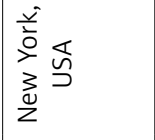 & 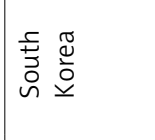 & 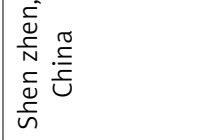 \\
\hline 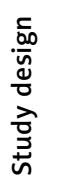 & 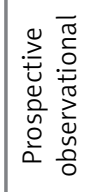 & 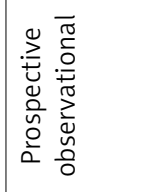 & 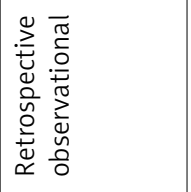 & 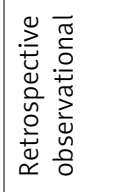 & 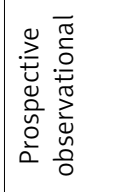 & 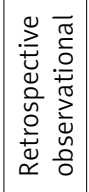 & 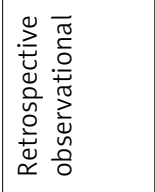 & 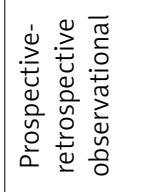 & 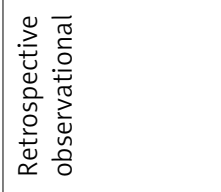 \\
\hline 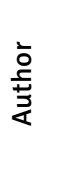 & 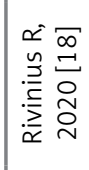 & 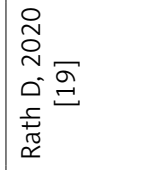 & 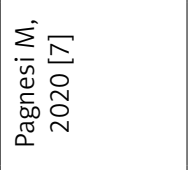 & 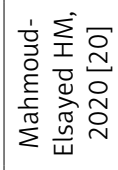 & $\begin{array}{l}\stackrel{0}{O} \\
\stackrel{N}{\Xi} \\
\stackrel{\Xi}{\Xi} \\
ت\end{array}$ & 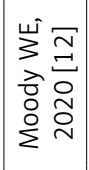 & 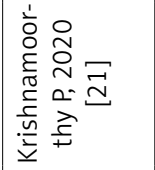 & 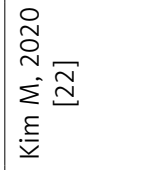 & 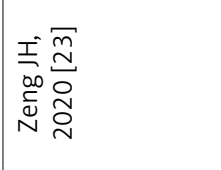 \\
\hline$\dot{\dot{z}}$ & $\rightarrow$ & $\sim$ & $m$ & t & in & 0 & $r$ & $\infty$ & $a$ \\
\hline
\end{tabular}




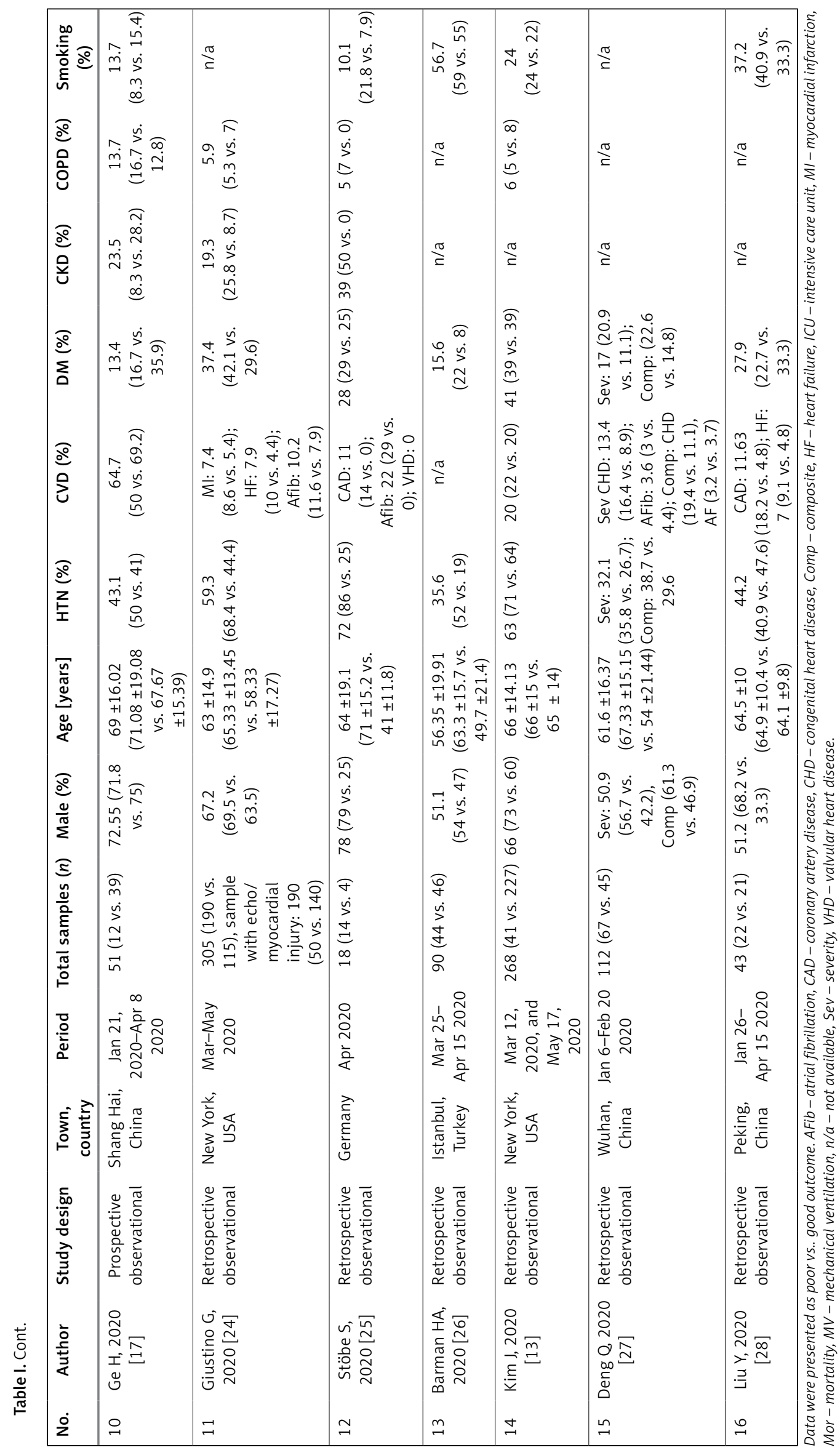


Table SI. Echocardiographic parameters of included studies are described in Supplementary Table SII. Eleven studies were retrospective, and five studies were prospective observational. One study was published in the preprint server [17]. Most studies were conducted in China. Tricuspid annular plane systolic excursion (TAPSE) and pulmonary artery systolic pressure (PASP) were used to define RVD and $\mathrm{PH}$, respectively, in most of the included studies.

\section{Quality assessment and publication bias}

Quality of the total included studies showed good and fair methodology based on NOS assessment (Supplementary Table SI). However, most studies did not assess exposure before outcome measures and might not have adequate timeframes for outcome due to their cross-sectional design.

Grading of Recommendations Assessment, Development, and Evaluation (GRADE) showed a very low certainty of evidence for the effect of RVD on mortality and MV usage; and low certainty of evidence on severity and ICU admission, while the effect of $\mathrm{PH}$ on mortality demonstrated high certainty of evidence and moderate certainty on severity, ICU admission, and MV usage, respectively (Supplementary Table SIII).

Funnel plots of RVD and outcome of mortality, severity, and MV showed a qualitatively asymmet- rical appearance indicating publication bias, but not for ICU outcome (Supplementary Figure S1). Funnel plots of $\mathrm{PH}$ and outcome of severity and ICU admission also showed a qualitatively asymmetrical appearance indicating publication bias, but not for mortality outcome (Supplementary Figure S2). Regression-based Harbord's or Egger's test was not conducted due to the small number of included studies (<10 studies) in each outcome.

\section{Right ventricular dysfunction in COVID-19}

RVD and RV function echocardiography parameter data were reported in 16 studies. Most studies defined RVD by TAPSE < 16-17 mm. The studies by Rath et al. (2020) [19], Li et al. (2020) [11], and Krishnamoorthy et al. (2020) [21] define RVD by TAPSE < $20 \mathrm{~mm}$, RV free wall longitudinal strain (RVFWS) $\leq 20.5 \%$, and RV global longitudinal strain (RVGLS) - RVFWS criteria in the American Society of Echocardiography (ASE) guideline, respectively. Two studies specifically enrolled COVID-19 patients with heart transplant [18] and myocardial injury [24].

Pooled prevalence of RVD from 14 studies was $19 \%\left(95 \% \mathrm{Cl}: 13-25 \% ; l^{2}=90.74 \%, p<0.001\right)$ (Figure 2 A). However, analysis for $\mathrm{PH}$-matched studies (6 studies) showed that prevalence of RVD was $13 \%\left(95 \% \mathrm{Cl}: 6-19 \% ; l^{2}=83.75 \%, p<0.001\right)$ (Figure 2 B).

Rivinius R, 2020
Dominik R, 2020
Pagnesi M, 2020
Mahmoud-Elsayed HM, 2020
Li Y, 2020
Moody WE, 2020
Krishnamoorthy P, 2020
Zeng JH, 2020
Ge H, 2020
Giustino G, 2020
Barman HA, 2020
Kim J, 2020
Deng Q
Liu Y
Overall ( $\left.I^{2}=90.74 \%, p<0.001\right)$

$0.29(0.14,0.50) \quad 4.39$
$0.07(0.03,0.12) \quad 8.40$ $0.14(0.10,0.20) \quad 8.29$ $0.27(0.18,0.38) \quad 6.91$ $0.33(0.26,0.41) \quad 7.65$ $0.35(0.28,0.43) \quad 7.71$ $\begin{array}{ll}0.42(0.19,0.68) & 2.84 \\ 0.05(0.02,0.14) & 8.09\end{array}$ $0.16(0.08,0.28) \quad 6.95$ $0.21(0.16,0.26) \quad 8.36$ $0.17(0.10,0.26) \quad 8.36$ $0.15(0.11,0.20) \quad 8.41$ $\begin{array}{ll}0.04(0.01,0.09) & 8.55 \\ 0.30(0.19,0.45) & 5.84\end{array}$ $\begin{array}{ll}0.30(0.19,0.45) & 5.84 \\ 0.19(0.13,0.25) & 100.00\end{array}$

B

RVD prevalence in COVID-19 (matched PH)

Study ES $(95 \% \mathrm{Cl}) \quad$ Weight $(\%)$

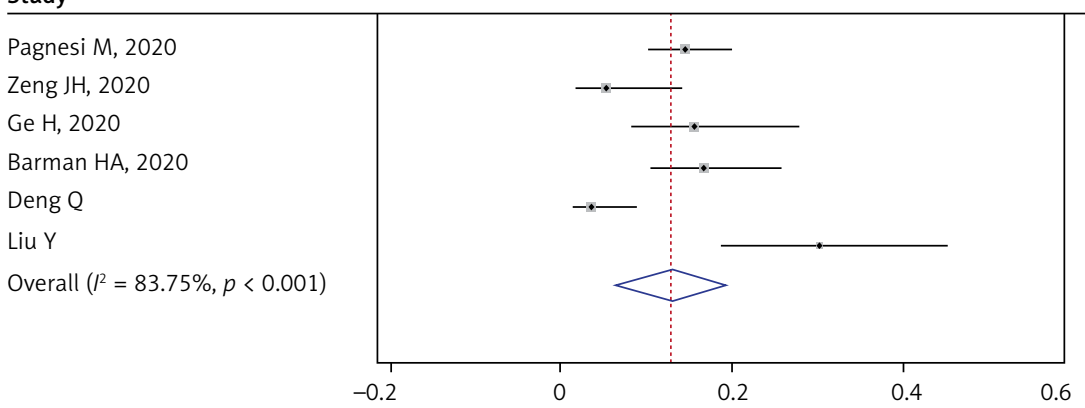

Figure 2. RVD prevalence in COVID-19 patients: total included studies (A) and PH-matched studies (B) 
Mortality outcome was analyzed from 8 studies and 965 participants. Pooled analysis with random effects showed that the RVD group had a higher mortality rate compared to the non-RVD group $(\mathrm{OR}=2.98(95 \% \mathrm{Cl}: 1.50-5.89), p=0.002$ $p^{2}=67 \%, p=0.004$ ) (Figure $3 \mathrm{~A}$ ). A sensitivity analysis by removing the study by Li et al. (2020) [11] or Liu et al. (2020) [28] exhibited a consistent result with lower heterogeneity $(\mathrm{OR}=2.55$ (95\% Cl: $1.27-5.13), p=0.009 ; P^{2}=63 \%, p=0.01$; and $(\mathrm{OR}=2.51(95 \% \mathrm{Cl}: 1.31-4.81), p=0.005$; $R^{2}=63 \%, p=0.01$, respectively). Subsequently, when a pre-printed study was removed by sensitivity analysis, the overall outcomes still showed a significant result with lower $\mathrm{OR}(\mathrm{OR}=2.65$ (95\% Cl: $1.31-5.36), p=0.007 ; l^{2}=67 \%, p=0.006$ ).

Random-effects meta-regression analysis demonstrated that the association between RVD and increased mortality was not significantly affected by age $(p=0.065)$, HTN ( $p=0.865)$, CVD $(p=0.206), \operatorname{CKD}(p=0.552), \operatorname{COPD}(p=0.839)$ comorbidities, and smoking $(p=0.561)$, but was significantly affected by male sex $(p=0.036)$, VTE $(p=0.044)$, and DM ( $p=0.001)$ (Supplementary Figure S3).

RV function echocardiographic parameters, TAPSE and RV fractional area change (RVFAC), were evaluated in 3 studies. TAPSE were significantly lower in the non-survivor group using random-effects analysis (MD $=-3.38(95 \% \mathrm{Cl}:-5.87$ to -0.89 ) $p=0.008 ; p^{2}=79 \%, p=0.008$ ) (Figure 3 B). Removing the study by Liu et al. [28] gave the same result with reduced heterogeneity $(\mathrm{MD}=-2.16(95 \% \mathrm{Cl}:-3.60$ to -0.71$) p=0.003$; $\left.p^{2}=0 \%, p=0.91\right)$. Similarly, RVFAC were lower in the non-survivor group with low heterogeneity $(\mathrm{MD}=-5.75 \%(95 \% \mathrm{Cl}:-8.23$ to -3.26$) p=0.001$; $P^{2}=0 \%, p=0.41$ ) (Figure $3 \mathrm{C}$ ).

Pooled analysis of 4 studies showed that a more severe clinical presentation was observed in the RVD group using fixed-effect analysis $\left(\mathrm{OR}=3.61(95 \% \mathrm{Cl}: 2.05-6.35), p<0.001 ; l^{2}=0 \%\right.$, $p=0.53$ ) (Supplementary Figure S4 A). Random-effects meta-regression analysis demonstrated that

A

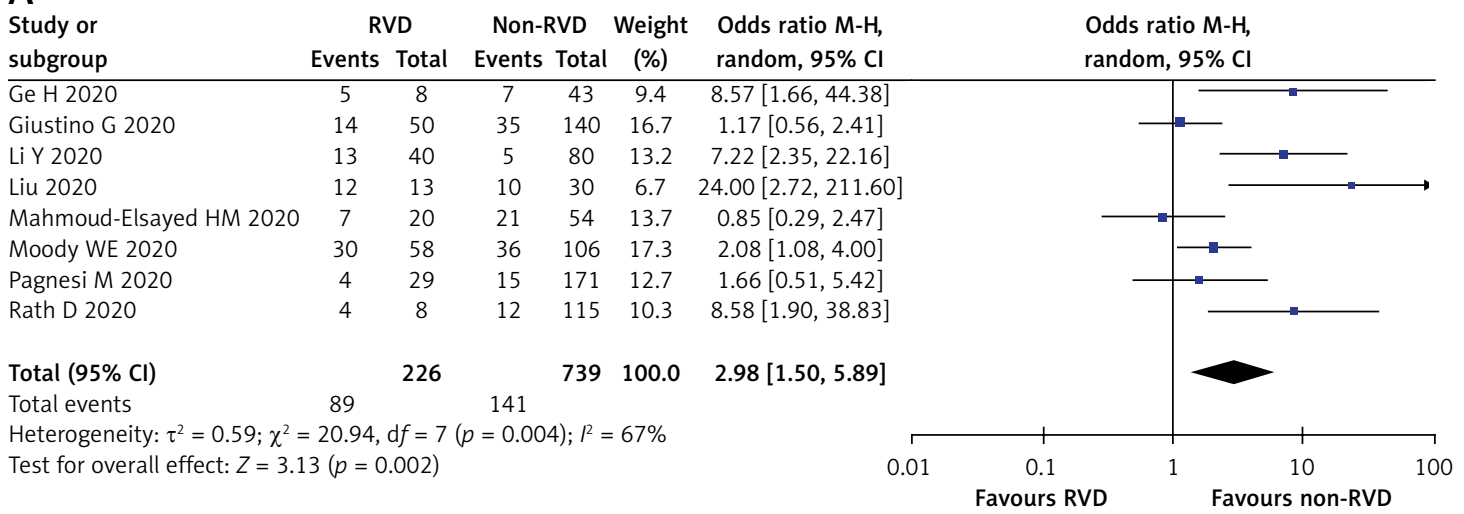

\section{B}

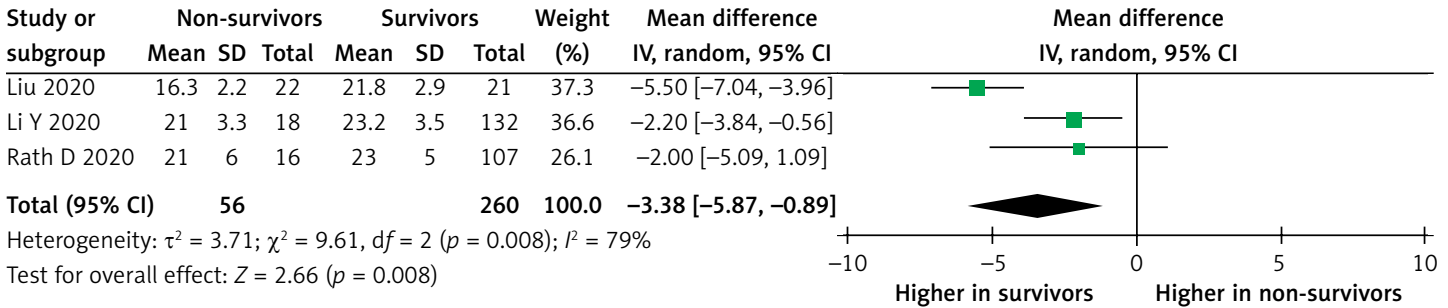

C

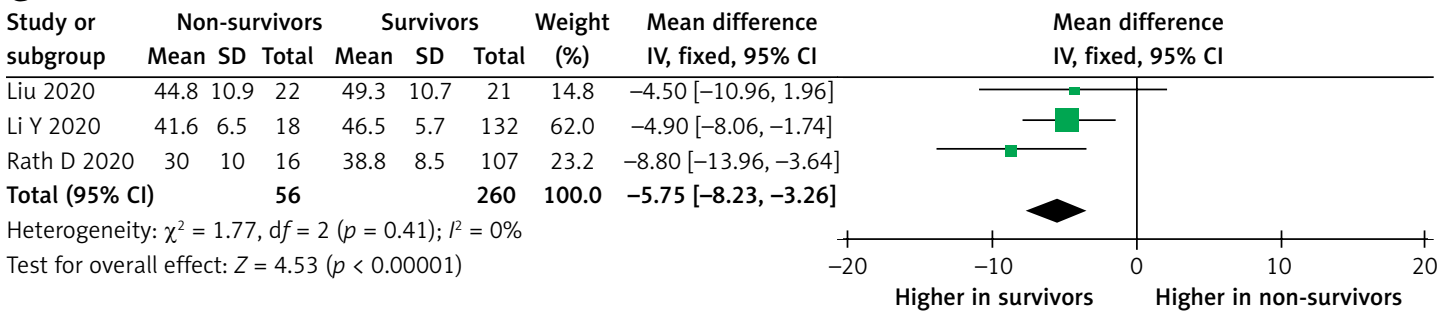

Figure 3. A - Mortality rate in COVID-19 patients with RVD. Comparison of RV function echocardiographic parameters: TAPSE (B) and RVFAC (C) in survivors vs. non-survivors

$R V$-right ventricle, $R V D-R V$ dysfunction, $R V F A C-R V$ fractional area change, TAPSE - tricuspid annular plane systolic excursion. 
the association between RVD and increased severity was not significantly affected by male sex $(p=0.595)$, age $(p=0.699)$, HTN $(p=0.741), \mathrm{CVD}$ $(p=0.179)$, and DM $(p=0.925)$ comorbidities.

Additionally, various echocardiographic parameters were analyzed. Evaluation of TAPSE was performed in 3 studies with a total of 220 patients and values were significantly lower in a severe condition ( $\mathrm{MD}=-1.30$ (95\% Cl: -2.03 to -0.57$)$, $p=0.0005 ; l^{2}=0 \%, p=0.68$ ) (Supplementary Figure $4 \mathrm{~B}$ ). RVGLS was evaluated in 2 studies and was significantly higher in severe disease $(M D=$ 3.33 (95\% Cl: 0.85-5.82), $p=0.009 ; l^{2}=0 \%, p=$ 0.39) (Supplementary Figure S4 C). Both RVFAC and RV S' were not statistically different in both groups ( $\mathrm{MD}=-1.48 \%(95 \% \mathrm{Cl}:-4.33 \%$ to $1.36 \%)$, $p=0.31 ; p^{2}=0 \%, p=0.82 ;$ and $M D=-0.51(95 \%$ $\mathrm{Cl}:-1.16$ to 0.14$), p=0.12 ; l^{2}=0 \%, p=0.73$, respectively) (Supplementary Figures S4 D, E).

ICU admission was analyzed from a total of 311 patients from 5 studies. Overall, COVID-19 patients with RVD had higher ICU admission $\left(\mathrm{OR}=1.70(95 \% \mathrm{Cl}: 1.12-2.56), p=0.01 ; l^{2}=0 \%\right.$, $p=0.69)$ (Supplementary Figure S5). However, removing the study by Giustino et al. [24] demonstrated no difference between groups (OR $=1.49$ (95\% Cl: 0.88-2.535), $p=0.14 ; l^{2}=0 \%, p=0.65$ ). Analysis of echocardiographic parameters was not performed due to limited study data.

Random-effects meta-regression analysis demonstrated that the association between RVD and ICU admission was not significantly affected by male sex $(p=0.631)$, age $(p=0.196)$, HTN $(p=0.455), \operatorname{CVD}(p=0.970), \mathrm{DM}(p=0.567), \mathrm{CKD}$ $(p=0.938)$, COPD $(p=0.346)$, or smoking ( $p=$ 0.450).

Seven studies evaluated RVD and MV use. Pooled analysis using a random-effects model revealed higher mechanical ventilation use in RVD groups (OR $=1.60(95 \% \mathrm{Cl}: 1.14-2.25), p=$ $0.007 ; R^{2}=51 \%, p=0.06$ ) (Supplementary Figure S6). However, after excluding the study by $\mathrm{Li}$ et al. [11], there was no difference between groups $\left(\mathrm{OR}=1.40\right.$ (95\% Cl: 0.97-2.01), $p=0.07 ; l^{2}=34 \%$, $p=0.18)$, indicating a lack of statistical robustness. Moreover, removing participants with heart transplant [18] and current myocardial injury [24] also showed no difference $(\mathrm{OR}=1.33(95 \% \mathrm{Cl}$ : $\left.0.89-2.00), p=0.17 ; l^{2}=42 \%, p=0.14\right)$. Analysis of echocardiographic parameters was not performed due to limited study data.

Random-effects meta-regression analysis demonstrated that the association between RVD and need for MV was not significantly affected by male sex $(p=0.089)$, age $(p=0.422)$, HTN $(p=$ $0.519), \mathrm{CVD}(p=0.077), \mathrm{DM}(p=0.114), \mathrm{COPD}$ $(p=0.913)$, or smoking $(p=0.303)$, but was affected by CKD $(p=0.048)$ and VTE $(p=0.011)$ comorbidities.

\section{Pulmonary hypertension in COVID-19}

Pulmonary hypertension (PH) in COVID-19 patients was reported in nine studies. Most of the studies defined PH as PASP > 35-40 mm Hg. A pooled analysis from six studies showed that the prevalence of $\mathrm{PH}$ in COVID-19 was $22 \%$ (95\% Cl: $\left.14-31 \% ; P^{2}=83.02 \%, p<0.001\right)$, as shown in Figure 4.

A total of 294 COVID-19 patients with $\mathrm{PH}$ in three studies demonstrated the mortality outcome. A pooled analysis using a fixed-effect model showed that $\mathrm{PH}$ was significantly associated with increased risk of mortality $(\mathrm{OR}=5.42$ (95\% Cl: 2.66-11.06), $p<0.001 ; l^{2}=0 \%, p=0.77$ ), as shown in Figure $5 \mathrm{~A}$. Sensitivity analysis using the leave-one-out method showed no difference in heterogeneity; likewise, when the pre-printed study by Ge et al. [17] was removed, the overall result showed increase mortality $(\mathrm{OR}=5.75(95 \% \mathrm{Cl}$ : 2.49-13.27), $\left.p<0.0001 ; l^{2}=0 \%, p=0.52\right)$. Furthermore, a random-effect meta-analysis of $\mathrm{PH}$ echocardiographic parameters found that pulmonary artery systolic pressure (PASP) was significantly higher in the $\mathrm{PH}$ group (MD = 15.23 (95\% Cl: 7.8022.66), $p<0.0001 ; I^{2}=53 \%, p=0.15$ ) (Figure $5 \mathrm{~B}$ ).

Random-effects meta-regression analysis demonstrated that the association between $\mathrm{PH}$ and mortality was not significantly affected by male sex $(p=0.803)$, age $(p=0.677)$, $\operatorname{HTN}(p=$ $0.504), \operatorname{CVD}(p=0.691), \operatorname{DM}(p=0.817), \operatorname{CKD}(p=$ $0.589)$, COPD ( $p=0.589)$, or smoking $(p=0.777)$.

$\mathrm{PH}$ prevalence in COVID-19

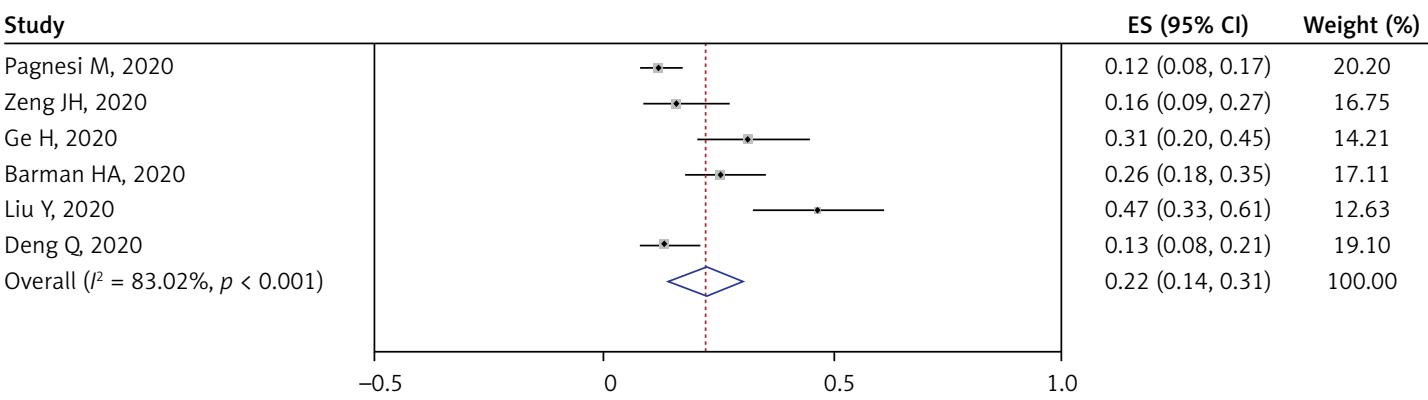

Figure 4. $\mathrm{PH}$ prevalence in COVID-19 patients. $\mathrm{PH}$ : pulmonary hypertension 
A

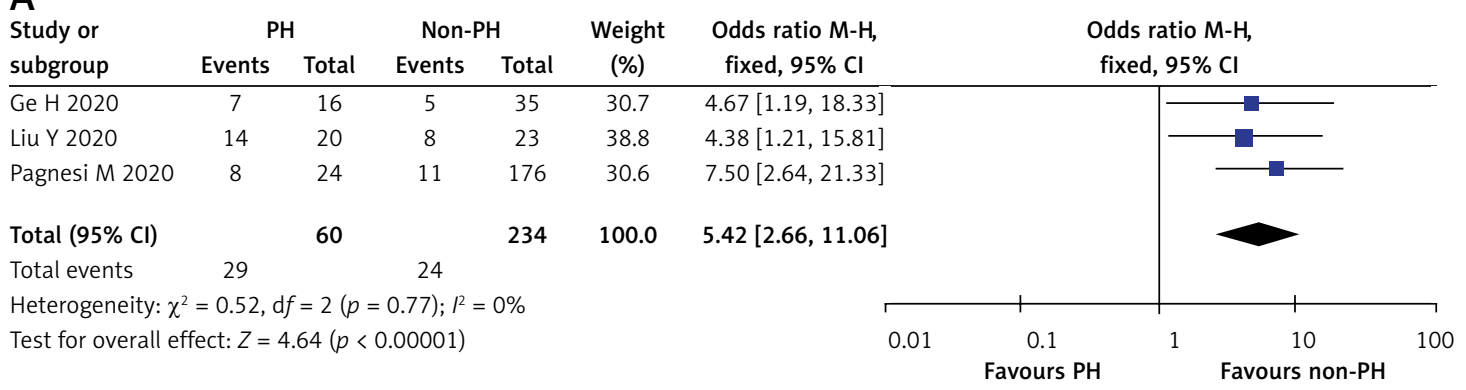

B

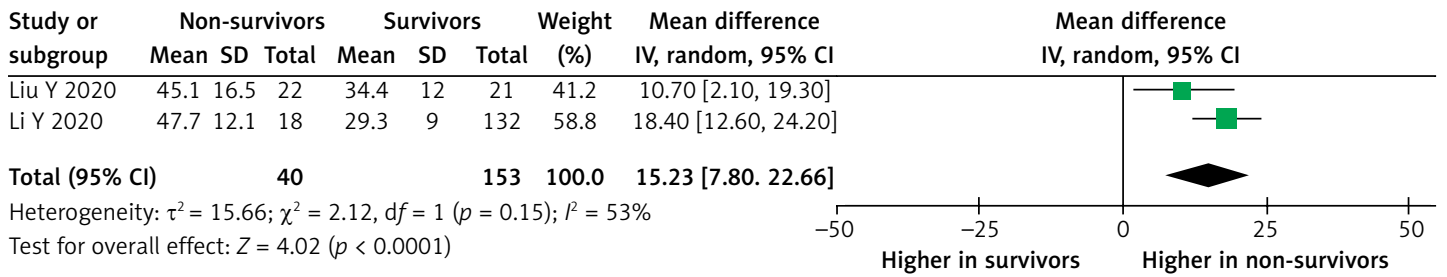

Figure 5. A - Mortality rate in COVID-19 patients with PH. B - Comparison of PASP in survivors vs. non-survivors

$P A S P$ - pulmonary artery systolic pressure, $\mathrm{PH}-$ pulmonary hypertension.

202 patients pooled from two studies [26, 27] were included to be analyzed. As shown in Supplementary Figure S7, the fixed-effect model showed that the $\mathrm{PH}$ group presented with more severe outcomes in COVID-19 patients (OR $=5.74$ (95\% Cl: $2.28-14.49), p<0.001 ; l^{2}=0 \%, p=0.37$ ). Interestingly, random-effect analysis revealed that the PASP between the two groups was not significantly different ( $\mathrm{MD}=3.24$ (95\% Cl: $-1.60-8.07)$, $\left.p=0.19 ; R^{2}=73 \%, p=0.02\right)$. Sensitivity analysis demonstrated reduced heterogeneity when the Barman et al. study [26] was removed from the pooled analysis (MD $=0.90(95 \% \mathrm{Cl}:-1.96-3.760$, $\left.p=0.54 ; l^{2}=0 \%, p=0.84\right)$.

ICU admission data were reported in two studies. The incidence of ICU admission is presented in Supplementary Figure S8. The fixed-effect model showed that $\mathrm{PH}$ was associated with increased incidence of ICU admission (OR $=12.83(95 \% \mathrm{CI}$ : 3.55-46.41), $\left.p<0.001 ; P^{2}=0 \%, p=0.52\right)$.

\section{Discussion}

The results of our meta-analysis showed that both RVD and PH in COVID-19 patients were associated with increased mortality, severity, ICU admission, and MV usage. Meta-regression revealed that only male sex significantly affected mortality in RVD, but not in $\mathrm{PH}$. Other comorbidities such as HTN, CVD, DM, CKD, COPD, and smoking did not affect the aforementioned outcomes. It is worth noting that the heterogeneity of our analysis for the effect estimates was moderate, and the certainty of the evidence was moderate to high in $\mathrm{PH}$ outcomes. Nevertheless, due to serious risk of bias in RVD outcomes, the certainty of the evidence remains low. To the best of our knowledge, our findings represent the most recent evidence of RVD and PH in COVID-19 patients along with their association with various clinical outcomes in one study.

Cardiac involvement in COVID-19 is well recognized. Interestingly, earlier studies demonstrated that RV abnormalities were more common compared to LV. A study from Wuhan in early 2020 reported that decreased RVFWS was found in $55.8 \%$, while decreased left ventricle (LV) global longitudinal strain (GLS) was found in $34.9 \%$. Additionally, patients with TAPSE $<17 \mathrm{~mm}$ were slightly more frequent than decreased LV ejection fraction (LVEF) findings [28]. Another study in TelAviv reported that $39 \%$ of hospitalized COVID-19 patients had RV dilatation or dysfunction, while only $10 \%$ and $16 \%$ of patients had LV systolic and diastolic dysfunction, respectively [29].

Mechanisms related to RVD in COVID-19 are unclear [30]. Numerous mechanisms are hypothetically possible in development of RV abnormalities. First, a possible mechanism is increased RV afterload. The hypercoagulability state in COVID-19 increases the occurrence of pulmonary thromboembolism and acutely increases RV afterload due to pulmonary hypoxic vasoconstriction. Modulation of ACE2 by SARS-CoV-2 is also predicted to alter pulmonary hemodynamics, raising pulmonary vascular resistance [30]. Respiratory distress and MV usage in COVID-19 might also increase RV afterload, therefore contributing to RVD development [31]. Another possible mechanism is cardiac injury. Cardiac injury in COVID-19 often affects the RV and spares the LV [29]. Injury in RV logically explains high-sensitive troponin elevation, despite normal LV function [12]. Finally, the ischemic con- 
dition from the LV or vascular dysfunction possibly influences RV performance [30].

Despite its unclear mechanism, RVD is often associated with poor clinical outcomes in COVID-19. One study claimed that RV echocardiographic findings provide better risk stratification than conventional risk factors. A univariable Cox model showed that RV dysfunction and RV dilatation were better in predicting all-cause mortality compared to other risk factors, including LV echocardiographic findings $(\mathrm{HR}=2.57(95 \% \mathrm{Cl}: 1.49-4.43)$, $p=0.001$; and $\mathrm{HR}=1.43(95 \% \mathrm{Cl}: 1.05-1.96), p=$ 0.02 , respectively). Additionally, combining either RV dysfunction or dilatation provides a comparable hazard ratio to $\mathrm{RV}$ dysfunction alone $(\mathrm{HR}=$ 2.76 (95\% Cl: 1.73-4.39), $p<0.001$ ) [13]. Similarly, a study in England demonstrated RVD outperforming conventional risk factors, namely sex, HTN, or diabetes, in predicting all-cause of mortality $(\mathrm{HR}=$ 1.80 (95\% Cl: $1.05-3.09), p=0.032)$. Furthermore, the same study confirmed that RVD could be used universally in all races, including Black, Asian, and other minority patients [12].

Contrary to our result, a study by Pagnesi et al. demonstrated that RVD was not associated with in-hospital all-cause mortality or ICU admission (log-rank $p$-value $=0.464)$ [7]. That study explained that $\mathrm{PH}$ is better in evaluating COVID-19 related hemodynamic changes, specifically in non-critically ill populations, which moderate changes in pulmonary artery pressure and are inadequate to cause secondary RV dysfunction. Further, RVD might be more related to MV usage.

Our results showed that RV parameters such as TAPSE, RVFAC, and RVGLS were significantly different in patients with poor clinical outcomes. This analysis confirms the study by Li et al. showing decreased TAPSE, RVFAC, and RVLS in the non-survivor group [11]. Moreover, analysis of the receiver-operating characteristic curve showed that RVLS had the highest performance for poor clinical outcome, followed by RVFAC and TAPSE. Yet, the optimal cut-off values were higher than the current guideline.

SARS-CoV-2 binds to ACE2 receptors, which are highly distributed in the lung and alters its function, leading to lung dysfunction, including $\mathrm{PH}$. The exact pathomechanism and association of $\mathrm{PH}$ with poor outcomes in COVID-19 patients need to be elucidated. Of the different investigated pathways, there are several potential mechanisms of $\mathrm{PH}$ in COVID-19. In response to lung injury, especially due to SARS CoV-2 infection, there is an abundance of evidence showing that the ACE2 receptor, as the main port of entry by the virus, is known to be down-regulated in $\mathrm{PH}$ [32]. As a consequence, the concentration of angiotensin II, which contributes to lung inflammation and inju- ry, tends to be elevated, and the protective role of ACE2 by converting angiotensin II to angioten$\sin (1-7)$ is diminished $[32,33]$. At the same time, elevated endothelin-1 in $\mathrm{PH}$ also down-regulated ACE2, inducing further vasoconstriction [34]. Intriguingly, the hypercoagulability and inflammatory state in COVID-19 promote the development of VTE and microthrombi that obstruct the pulmonary vessel, leading to $\mathrm{PH}$ [35]. In the current cases, microvascular injury plays a key role in the development of PH in COVID-19 patients [36].

It is well known that COVID-19 causes multi-organ damage, and the presence of cardiac and lung injury may worsen the outcomes. Esposito et al. [37] reported that non-survivor groups in COVID-19 patients displayed elevated PASP compared to survivor groups. This study also revealed that $\mathrm{PH}$, as diagnosed by enlarged main pulmonary artery diameter, was a predictor of mortality $(\mathrm{HR}(95 \% \mathrm{Cl})$ : 1.741 (1.253-2.418), $p<0.001)$. Likewise, our mortality was higher in $\mathrm{PH}$ groups. Moreover, the prevalence of $\mathrm{PH}$ in patients who develop cardiac injury is higher compared to non-cardiac injury [26].

In fact, PH and RV dysfunction in COVID-19 is part of a vicious cycle and associated with worse conditions. An increase in pulmonary artery pressure resulted in an increase in $\mathrm{RV}$ afterload that may continue to RV dysfunction [26]. In addition, ARDS as a complication in COVID-19 leads to respiratory failure that needs MV application, and such MV further increases PASP and exacerbates RV dysfunction. In line with our findings, a study in Europe showed that $66.67 \%$ of patients admitted to the ICU required $M V$, and the overall mortality rate in $\mathrm{PH}$ was $20 \%$ [38]. Another retrospective observational study previously reported that $63.6 \%$ of the total samples were admitted to the ICU, whereas $28.57 \%$ of them underwent MV [39]. This study also reported that the mortality rate among $\mathrm{PH}$ patients was $36.36 \%$, directly attributable to COVID-19. The authors revealed that systemic hypertension and diabetes mellitus were associated with severity of the disease; however, our meta-regression proved that these comorbidities were not significantly affected.

\section{Clinical implications and study limitations}

This study provides evidence regarding the importance of RVD and PH in COVID-19. RVD and PH parameters could be evaluated easily by bedside transthoracic echocardiography. These parameters could be developed as novel risk-stratification markers in COVID-19 patients since they outperformed conventional risk factors in previous evidence $[12,13]$. This study also supports experts' statements and consensus on the need for pointof-care ultrasound during the pandemic, which 
involves the measurement of right ventricle size and function, along with pulmonary artery systolic pressure [40-42]. In addition, evaluation in RVD and $\mathrm{PH}$ may provide a novel strategy and insights for COVID-19 management.

Nevertheless, our study has several limitations. Publication bias was noted in several outcomes. Most of the included studies in this meta-analysis were retrospective observational, with a relatively small sample size, and were not adequately matched/adjusted for confounders. Thus, the included studies were subject to potential confounders that may weaken or strengthen the effect estimate. The result of the meta-regression has to be interpreted cautiously due to the known limitations of such analysis. Definitions of RVD and PH were slightly different between studies. The definition of RVD was mostly based on TAPSE $<16 \mathrm{~mm}$ or TAPSE $<17 \mathrm{~mm}$, while most PH was defined based on PASP $>35 \mathrm{~mm} \mathrm{Hg}$ or PASP $>40 \mathrm{~mm} \mathrm{Hg}$. Yet, other variance in RVD and $\mathrm{PH}$ definition should not be neglected. Our study did not evaluate pre-existing RVD or $\mathrm{PH}$, which may cause a biased result. Lastly, despite the fact that RV's structural feature is not included in our objective, structural findings may reflect chronicity of RVD or $\mathrm{PH}$, and therefore may provide further information regarding its mechanism in COVID-19.

In conclusion, RVD and $\mathrm{PH}$ were prevalent in COVID-19 and associated with mortality, severity, ICU admission, and MV usage in COVID-19 patients. Bedside echocardiography examination could be considered as a novel risk stratification tool in COVID-19.

\section{Acknowledgments}

Trial Registry: UMIN Clinical Trial Registry (UMIN000042424).

\section{Conflict of interest}

The authors declare no conflict of interest.

\section{References}

1. WHO. WHO Coronavirus Disease (COVID-19) Dashboard.

2. Elsoukkary SS, Mostyka M, Dillard A, et al. Autopsy findings in 32 patients with COVID-19: a single-institution experience. Pathobiology 2021; 88: 56-68.

3. Li B, Yang J, Zhao F, et al. Prevalence and impact of cardiovascular metabolic diseases on COVID-19 in China. Clin Res Cardiol 2020; 109: 531-8.

4. Zheng YY, Ma YT, Zhang JY, et al. COVID-19 and the cardiovascular system. Nat Rev Cardiol 2020; 17: 259-60.

5. Santoso A, Pranata R, Wibowo A, et al. Cardiac injury is associated with mortality and critically ill pneumonia in COVID-19: a meta-analysis. Am J Emerg Med 2021; 44: 352-7.

6. Nishiga M, Wang DW, Han Y, et al. COVID-19 and cardiovascular disease: from basic mechanisms to clinical perspectives. Nature Rev Cardiol 2020; 17: 543-58.
7. Pagnesi M, Baldetti L, Beneduce A, et al. Pulmonary hypertension and right ventricular involvement in hospitalised patients with COVID-19. Heart 2020; 106: 1324-31.

8. Sylvester JT, Shimoda LA, Aaronson PI, et al. Hypoxic pulmonary vasoconstriction. Physiol Rev 2012; 92: 367-520.

9. Karmouty-Quintana H, Thandavarayan RA, Keller SP, et al. Emerging mechanisms of pulmonary vasoconstriction in SARS-CoV-2-induced acute respiratory distress syndrome (ARDS) and potential therapeutic targets. Int J Mol Sci 2020; 21: 1-21.

10. Creel-Bulos C, Hockstein M, Amin N, et al. Acute cor pulmonale in critically ill patients with Covid-19. N Engl J Med 2020; 382: e70.

11. Li Y, Li H, Zhu S, et al. Prognostic value of right ventricular longitudinal strain in patients with COVID-19. J Am Coll Cardiol 2020; 13: 2287-99.

12. Moody WE, Mahmoud-Elsayed HM, Senior J, et al. Impact of right ventricular dysfunction on mortality in patients hospitalized with COVID-19 according to race. CJC Open 2021; 3: 91-100.

13. Kim J, Volodarskiy A, Sultana R, et al. Prognostic utility of right ventricular remodeling over conventional risk stratification in patients with COVID-19. J Am Coll Cardiol 2020; 76: 1965-77.

14. World Health Organization. Report of the WHO-China Joint Mission on Coronavirus Disease 2019 (COVID-19).

15. Wan X, Wang W, Liu J, Tong. Estimating the sample mean and standard deviation from the sample size, median, range and/or interquartile range. BMC Med Res Methodol 2014; 14: 135.

16. Higgins J, Thomas J, Chandler J, et al. Cochrane Handbook for Systematic Reviews of Interventions version 6.0 (updated July 2019). Cochrane.

17. Ge H, Zhu M, Du J, et al. Cardiac structural and functional characteristics in patients with coronavirus disease 2019: a serial echocardiographic study. medRxiv 2020; 2020.05.12.20095885.

18. Rivinius R, Kaya Z, Schramm R, et al. COVID-19 among heart transplant recipients in Germany: a multicenter survey. Clin Res Cardiol 2020; 109: 1531-9.

19. Rath D, Petersen-Uribe Á, Avdiu A, et al. Impaired cardiac function is associated with mortality in patients with acute COVID-19 infection. Clin Res Cardiol 2020; 109: 1491-9.

20. Mahmoud-Elsayed HM, Moody WE, Bradlow WM, et al. Echocardiographic findings in patients with COVID-19 pneumonia. Can J Cardiol 2020; 36: 1203-7.

21. Krishnamoorthy P, Croft LB, Ro R, et al. Biventricular strain by speckle tracking echocardiography in COVID-19: findings and possible prognostic implications. Future Cardiol 2021; 17 DOI: 10.2217/fca-2020-0100.

22. Kim M, Nam JH, Son JW, et al. Cardiac manifestations of coronavirus disease 2019 (COVID-19): a multicenter cohort study. J Korean Med Sci 2020; 35: e366.

23. Zeng JH, Wu WB, Qu JX, et al. Cardiac manifestations of COVID-19 in Shenzhen, China. Infection 2020; 48: 861-70.

24. Giustino G, Croft LB, Stefanini GG, et al. Characterization of myocardial injury in patients with COVID-19. J Am Coll Cardiol 2020; 76: 2043-55.

25. Stöbe S, Richter S, Seige $M$, et al. Echocardiographic characteristics of patients with SARS-CoV-2 infection. Clin Res Cardiol 2020; 109: 1549-66.

26. Barman HA, Atici A, Tekin EA, et al. Echocardiographic features of patients with COVID-19 infection: a crosssectional study. Int J Cardiovasc Imaging 2021; 37: 825-34. 
27. Deng Q, Hu B, Zhang Y, et al. Suspected myocardial injury in patients with COVID-19: evidence from front-line clinical observation in Wuhan, China. Int J Cardiol 2020; 311: 116-21.

28. Liu Y, Xie J, Gao P, et al. Swollen heart in COVID-19 patients who progress to critical illness: a perspective from echo-cardiologists. ESC Hear Fail 2020; 7: 3621-32.

29. Szekely Y, Lichter Y, Taieb P, et al. Spectrum of cardiac manifestations in COVID-19: a systematic echocardiographic study. Circulation 2020; 142: 342-53.

30. Park JF, Banerjee S, Umar S. In the eye of the storm: the right ventricle in COVID-19. Pulm Circ 2020; 10: 2045894020936660.

31. Repessé X, Vieillard-Baron A. Right heart function during acute respiratory distress syndrome. Ann Trans Med 2017; 5: 295.

32. Farha S. COVID-19 and pulmonary hypertension. Cleve Clin J Med 2020. DOI: 10.3949/ccjm.87a.ccc021.

33. Jia H. Pulmonary angiotensin-converting enzyme 2 (ACE2) and inflammatory lung disease. Shock 2016; 46 : 239-48.

34. Zhang H, Li Y, Zeng Y, et al. Endothelin-1 downregulates angiotensin-converting enzyme-2 expression in human bronchial epithelial cells. Pharmacology 2013; 91: 297-304.

35. Wichmann D, Sperhake JP, Lütgehetmann M, et al. Autopsy findings and venous thromboembolism in patients with COVID-19: a prospective cohort study. Ann Intern Med 2020; 173: 268-77.

36. van Dongen CM, Janssen MT, van der Horst RP, et al. Unusually rapid development of pulmonary hypertension and right ventricular failure after COVID-19 pneumonia. Eur J Case Rep Intern Med 2020; 7: 001784.

37. Esposito A, Palmisano A, Toselli M, et al. Chest CT-derived pulmonary artery enlargement at the admission predicts overall survival in COVID-19 patients: insight from 1461 consecutive patients in Italy. Eur Radiol 2021; 31: 4031-41.

38. Belge C, Quarck R, Godinas L, et al. COVID-19 in pulmonary arterial hypertension and chronic thromboembolic pulmonary hypertension: a reference centre survey. ERJ Open Res 2020; 6: 00520-2020.

39. Sulica R, Cefali F, Motschwiller C, et al. Diagnostics COVID-19 in pulmonary artery hypertension (PAH) patients: observations from a large PAH Center in New York City. Diagnostics 2021; 11: 128.

40. Johri AM, Galen B, Kirkpatrick JN, et al. ASE Statement on point-of-care ultrasound during the 2019 novel coronavirus pandemic. J Am Soc Echocardiogr 2020; 33: 670-3.

41. Hussain A, Via G, Melniker L, et al. Multi-organ point-ofcare ultrasound for COVID-19 (PoCUS4COVID): international expert consensus. Crit Care 2020; 24: 702.

42. Agricola E, Beneduce A, Esposito A, et al. Heart and lung multimodality imaging in COVID-19. JACC CardiovasC Imaging 2020; 13: 1792-808. 